\title{
Dietary Polyphenol Intake Is Associated with Biological Aging, a Novel Predictor of Cardiovascular Disease: Cross-Sectional Findings from the Moli-Sani Study
}

\author{
Simona Esposito ${ }^{1}$, Alessandro Gialluisi ${ }^{1}$ (D), Simona Costanzo ${ }^{1}$, Augusto Di Castelnuovo ${ }^{2}$, Emilia Ruggiero ${ }^{1}$ (D), \\ Amalia De Curtis ${ }^{1}$ (D), Mariarosaria Persichillo ${ }^{1}$, Chiara Cerletti ${ }^{1} \mathbb{D}_{\text {, Maria Benedetta Donati }}{ }^{1}$, \\ Giovanni de Gaetano ${ }^{1}$ D , Licia Iacoviello ${ }^{1,3, * \mathbb{D}}$, Marialaura Bonaccio ${ }^{1}$ and on behalf of the Investigators \\ for the Moli-Sani Study ${ }^{\dagger}$
}

1 Department of Epidemiology and Prevention, IRCCS Neuromed, via dell'Elettronica, 86077 Pozzilli, Italy; simona.esposito@moli-sani.org (S.E.); alessandro.gialluisi@moli-sani.org (A.G.); simona.costanzo@moli-sani.org (S.C.); emilia.ruggiero@moli-sani.org (E.R.); amalia.decurtis@moli-sani.org (A.D.C.); mariarosaria.persichillo@moli-sani.org (M.P.); chiara.cerletti@moli-sani.org (C.C.); mbdonati@moli-sani.org (M.B.D.); giovanni.degaetano@moli-sani.org (G.d.G.); marialaura.bonaccio@moli-sani.org (M.B.)

2 Mediterranea Cardiocentro, 80122 Napoli, Italy; dicastel@ngi.it

check for updates

Citation: Esposito, S.; Gialluisi, A.; Costanzo, S.; Di Castelnuovo, A.;

Ruggiero, E.; De Curtis, A.;

Persichillo, M.; Cerletti, C.; Donati, M.B.; de Gaetano, G.; et al. Dietary Polyphenol Intake Is Associated with Biological Aging, a Novel Predictor of Cardiovascular Disease: Cross-

Sectional Findings from the Moli-Sani Study. Nutrients 2021, 13, 1701. https://doi.org/10.3390/ nu13051701

Academic Editors: Assunta Pandolfi and Domitilla Mandatori

Received: 13 April 2021

Accepted: 12 May 2021

Published: 17 May 2021

Publisher's Note: MDPI stays neutral with regard to jurisdictional claims in published maps and institutional affiliations.

Copyright: (c) 2021 by the authors. Licensee MDPI, Basel, Switzerland. This article is an open access article distributed under the terms and conditions of the Creative Commons Attribution (CC BY) license (https:// creativecommons.org/licenses/by/ $4.0 /)$.
3 Department of Medicine and Surgery, Research Center in Epidemiology and Preventive Medicine (EPIMED), University of Insubria, 21100 Varese-Como, Italy

* Correspondence: licia.iacoviello@moli-sani.org

+ Moli-sani Study investigators are provided in the Acknowledgments.

Abstract: Biological aging, or the discrepancy between biological and chronological age of a subject ( $\Delta$ age), has been associated with a polyphenol-rich Mediterranean diet and represents a new, robust indicator of cardiovascular disease risk. We aimed to disentangle the relationship of dietary polyphenols and total antioxidant capacity with $\Delta$ age in a cohort of Italians. A cross-sectional analysis was performed on a sub-cohort of 4592 subjects (aged $\geq 35 \mathrm{y} ; 51.8 \%$ women) from the Moli-sani Study (2005-2010). Food intake was recorded by a 188-item food-frequency questionnaire. The polyphenol antioxidant content (PAC)-score was constructed to assess the total dietary content of polyphenols. Total antioxidant capacity was measured in foods by these assays: trolox equivalent antioxidant capacity (TEAC), total radical-trapping antioxidant parameter (TRAP) and ferric reducing-antioxidant power (FRAP). A deep neural network, based on 36 circulating biomarkers, was used to compute biological age and the resulting $\Delta$ age, which was tested as outcome in multivariable-adjusted linear regressions. $\triangle$ age was inversely associated with the PAC-score $(\beta=-0.31 ; 95 \% C I-0.39,-0.24)$ but not with total antioxidant capacity of the diet. A diet rich in polyphenols, by positively contributing to deceleration of the biological aging process, may exert beneficial effects on the long-term risk of cardiovascular disease and possibly of bone health.

Keywords: biological aging; dietary polyphenols; cardiovascular disease; bone health

\section{Introduction}

In the last century, the average life expectancy at birth has increased remarkably, from roughly 45 years in the early 1900s to approximately 80 years today. By 2050, over $21 \%$ of the global population will be over 60 years of age and this will lead to an increase in many age-related diseases and disabilities, with tremendous costs for public health systems [1-3].

Aging is characterized by a time-dependent functional decline that affects most living organisms [4], and is usually indicated by chronological age (CA) [5].

Recent studies proposed that CA can no longer sufficiently reflect the health status of individuals [6], and biological age (BA), the hypothetical underlying functional age of an organism, has gained increasing attention in recent years [7]. The assessment of 
BA is based on diverse parameters, e.g., blood biomarkers [8,9], DNA methylation [10], spirometry [11] and structural neuroimaging measures [12]. BA and CA may often differ within an organism and for this reason it is reasonable to compute a difference between BA and CA (hereafter called $\Delta$ age), which may be positive (suggesting accelerated biological aging) or negative (indicating decelerated biological aging) [7].

Indeed, observational studies showed that subjects with decelerated biological aging (i.e., BA $<<$ CA), have lower risk for morbidity, disability and mortality [13,14]. Additionally, recent epidemiological research suggested that $\Delta$ age may play a role in prediction of all-cause mortality [8,12] and hospitalization for cardiovascular causes [14]. Similarly, biological aging accounted for higher risk of all-cause and cause-specific mortality in non-Caucasian ancestries, e.g., African-Americans [15] and Koreans [16].

Therefore, among individuals with the same CA, a difference in measured BA would reflect differences in decreased or increased risk for age-related cardiovascular disease, disability and death.

Lifestyles such as smoking, physical activity and diet are among environmental factors that possibly influence biological aging $[14,17,18]$.

Prior work showed that a healthy diet, such as a Mediterranean diet [10], is associated with decelerated biological aging [10]. Our group recently reported evidence that subjects with high adherence to Mediterranean diet are on average almost 1 year biologically younger than their CA, as compared to those with low adherence [14].

A Mediterranean diet is traditionally rich in foods that are major sources of polyphenols, naturally occurring bioactive compounds that are the most abundant antioxidants in the diet [19].

In addition to being powerful antioxidants, polyphenols also possess anti-inflammatory properties, and their role in the prevention and treatment of various diseases linked to oxidative stress or inflammation, such as cardiovascular disease, has been extensively reported [20]. Also, polyphenol-rich diet are favourably associated with bone mineral density [21]; in particular, high consumption of extra-virgin olive oil, a major source of polyphenols, leads to lower risk of osteoporosis-related fractures [22].

To date, the association between polyphenol-rich diets and biological aging is not fully elucidated, although some data point to a favourable relationship between consumption of antioxidant-rich foods and some markers of aging, such as leukocyte mitochondrial DNA variations [23] and telomere attrition [23-25].

There is also a lack of knowledge on whether the total antioxidant capacity of the diet (TAC), which sums up the free radical scavenging ability of antioxidants in foods [26], is associated with biological aging.

In order to fill this knowledge gap, the present study sought to test the relationship between dietary polyphenols and the total antioxidant capacity of the diet with biological aging, using data from a large cohort of Italian adults.

\section{Materials and Methods}

\subsection{Study Population}

We analysed data from the Moli-sani Study, a large population-based cohort study designed to investigate genetic and environmental risk factors in the onset of cardiovascular, cerebrovascular and tumor diseases [27]. At the baseline survey performed between 2005 and 2010, 24,325 subjects (aged $\geq 35 \mathrm{y}$ ) were recruited from city-hall registries of the Molise region. Exclusion criteria were: pregnancy at the time of recruitment, disturbances in understanding or willingness, current poly-traumas or coma, or refusal to sign the informed consent form. The Moli-sani study complied with the Declaration of Helsinki and was granted the approval of the Ethics Committee of the Catholic University, Rome, Italy. Details of the study design are available elsewhere [27].

From the initial 24,325 participants, $\Delta$ age was calculated in a test set of 4772 subjects, as described below and elsewhere [14]. For the present analysis, we omitted individuals reporting dietary questionnaires judged as unreliable by the interviewers $(n=179)$, and 
participants with lack of information on diet $(n=20)$. The final analysed sample consisted of 4592 subjects.

\subsection{Computation of Biological Age}

To compute biological age, we used a supervised machine learning algorithm called a deep neural network (DNN), as described in [14]. Briefly, we deployed a DNN for the prediction of BA using 36 circulating biomarkers, recruiting centre and sex as input features, and CA of each participant as a label. Biomarkers used included: lipid biomarkers, including triglycerides, high and low density lipoprotein-cholesterol, lipoprotein a and apolipoprotein A1 and B; markers of glucose metabolism such as glucose, C-peptide and insulin; liver enzymes, aspartate transaminase and alanine aminotransferase; cardiac and vascular markers such as NT-proB-type Natriuretic Peptide and high-sensitivity cardiac troponin I; other hormones such as testosterone and vitamin D; haemostasis markers such as D-Dimer; renal markers (uric acid, albumin, creatinine, cystatin-C); inflammation markers such as high sensitivity C-reactive protein; common haemachrome markers, including red blood cell count and distribution width, haematocrit, haemoglobin levels, mean corpuscular volume, mean corpuscular haemoglobin concentration, total white blood cells, lymphocytes, monocytes, granulocytes, neutrophils, basophils and eosinophils; platelet count, mean platelet volume and platelet distribution width.

The DNN was built in R v3.9 (https: / / www.r-project.org/) through the Keras package v2.4.0 (https:// cran.r-project.org/web/packages/keras/index.html). Briefly, we split the available dataset passing QC $(N=23,858)$ into a random training and test set $(80: 20$ ratio), then trained the algorithm over 1000 epochs in the training set and evaluated the accuracy in the test set. Finally, BA for each participant and the resulting discrepancy with CA was computed $(\triangle \mathrm{age}=\mathrm{BA}-\mathrm{CA})$ within the test set $(N=4772)$, which was used as the population of study herein. Details on QC, DNN architecture and performance are reported in [14].

\subsection{Dietary Assessment}

Food intake during the past 12 months was assessed by the EPIC food frequency questionnaire (FFQ) [28] validated and adapted to the Italian population, for a total of 188 food items that were classified into 74 predefined food groups on the basis of similar nutrient characteristics or culinary usage.

Through the use of a specifically designed software [29], frequencies and quantities of each food were linked to Italian food tables [30] to obtain estimates of daily intake of macro- and micro-nutrients and energy, and integrated with the TAC values of several foods representative of the average Italian diet, such as fruits, vegetables, oils, beverages, spices, dried fruits, sweets, cereals, pulses and nuts [31].

Polyphenol antioxidant content (PAC) of the diet was appraised by a PAC-score as in Pounis et al. [32], a holistic approach that took into account the potential synergistic effects of polyphenols and likely lowered the possibility for biased regression estimations deriving from multi-correlation of nutritional data For each dietary source of polyphenols (i.e., vegetables, fruits, beverages and alcoholic beverages, for a total of 23 food groups), the mean content in different classes and subclasses of polyphenols was calculated according to the availability of food composition table data. Using this information and the daily consumption of each food source, the total intakes of seven classes and sub-classes of polyphenols were calculated as follows: flavonols $(\mathrm{mg} / \mathrm{d})$, flavones $(\mathrm{mg} / \mathrm{d})$, flavanones (mg/d), flavanols ( $\mathrm{mg} / \mathrm{d})$, anthocyanidins $(\mathrm{mg} / \mathrm{d})$, isoflavones $(\mathrm{mg} / \mathrm{d})$ and lignans $(\mathrm{mg} / \mathrm{d})$. The choice of units presented for polyphenols was done according to the units used in the original source [33].

Deciles of total intakes of each polyphenol class and sub-class were then generated; for all polyphenol components, higher intakes (that is, $>$ Q6) scored positively, while lower intakes (that is, $<\mathrm{Q} 5$ ) received negative scoring. The PAC-score ranged between -28 and 28 , and resulted from the sum of the seven components. An increase in the score indicated higher total content of polyphenols in the diet. 
TAC was measured in foods via the use of three different assays: the trolox equivalent antioxidant capacity (TEAC, mmol Trolox) assay, measuring the antioxidants' ability to reduce a radical cation in both lipophilic and hydrophilic conditions; the radical-trapping antioxidant parameter (TRAP, mmol Trolox) and ferric reducing-antioxidant power (FRAP, $\mathrm{mmol} \mathrm{Fe}^{2+}$ ) assays, evaluating the chain-breaking antioxidant potential and the reducing power of the sample, respectively [31]. The food frequency questionnaire was specifically validated for assessment of dietary TAC against TRAP, FRAP and TEAC values estimated by a 3-day weighed food record and plasma TEAC and FRAP in a group of healthy Italian adults [34].

\subsection{Ascertainment of Risk Factors}

Information on sociodemographic variables, lifestyles and clinical variables were obtained by interviewer-administered questionnaires.

Participants were considered to have hypertension, hyperlipidaemia or diabetes if they reported having been treated with disease-specific drugs.

Leisure-time physical activity for sport, walking and gardening was self-reported and dichotomized as $<30$ or $\geq 30 \mathrm{~min} / \mathrm{d}$. Height and weight were measured and body mass index (BMI) was calculated as $\mathrm{kg} / \mathrm{m}^{2}$, and then grouped into three categories as normal $\left(\leq 25 \mathrm{~kg} / \mathrm{m}^{2}\right)$, overweight $\left(>25\right.$ and $\left.<30 \mathrm{~kg} / \mathrm{m}^{2}\right)$ or obese $\left(\geq 30 \mathrm{~kg} / \mathrm{m}^{2}\right)$. Subjects were classified as never-smokers, current smokers or former smokers (reported not having smoked at all over the previous 12 months or more). Education was based on the highest qualification attained and was categorized as (1) up to lower secondary (approximately $\leq 8$ years of study), (2) upper secondary school (8-13 years of study) and (3) postsecondary education ( $>13$ years of study).

\subsection{Statistical Analysis}

Characteristics of the study population were presented as numbers and percentages, or mean values and standard deviation (SD) for continuous variables.

Multivariable-adjusted linear regression analysis (PROC REG in SAS) was used to estimate the relationship between dietary factors (independent variable) and $\Delta$ age (dependent variable) and results were expressed as regression coefficients $(\beta)$ with a $95 \%$ confidence interval (95\%CI). Distribution of missing values was as follows: educational level $(n=1)$, BMI $(n=4)$, smoking habit $(n=5)$, history of cardiovascular disease $(n=69)$, cancer $(n=19)$, diabetes $(n=65)$, hyperlipidaemia $(n=45)$, hypertension $(n=43)$ and menopausal status $(n=4)$. We used a multiple imputation technique (SAS PROC MI, followed by PROC MIANALYZE) to maximize data availability for all variables, avoid bias introduced by not-at-random missing (MNAR) data patterns and achieve robust results over different simulations ( $n=10$ imputed datasets).

We first tested association of the PAC-score with $\triangle$ age, and then with each polyphenol subgroup intake. Finally, we tested associations with dietary antioxidant capacity measures (TEAC, TRAP, FRAP). Statistical tests were two-sided, and $p$ values of less than 0.05 were considered to indicate statistical significance for the analysis of the PAC-score; for the test of different polyphenol categories and antioxidant capacities, the $\alpha$ threshold underwent Bonferroni correction according to the number of markers tested $(\alpha=0.007$ and 0.017 , respectively).

Each dietary indicator was scaled by its standard deviation so that regression coefficients indicated the variation in $\Delta$ age for 1 standard deviation change for each dietary indicator.

Based on previous literature and biological plausibility, two models were fitted: model 1 was adjusted for age, sex and energy intake (Kcal/d); model 2 was further controlled for education, leisure-time physical activity, smoking habit, BMI, CVD, cancer, diabetes, hypertension, hyperlipidaemia, menopausal status, hormone replacement therapy and dietary fibre $(\mathrm{g} / \mathrm{d})$. 
Analyses were performed separately by sex. Appropriate multiplicative terms for testing interaction were included in the multivariable models to test for a difference of effect of dietary polyphenols/TAC by gender.

Data analyses were generated using SAS/STAT software, version 9.4 (SAS Institute Inc., Cary, NC, USA).

\section{Results}

The analysed population consisted of 2381 women (51.8\%) and 2211 men (48.2\%) with a mean CA of $55.6 \mathrm{y}( \pm 11.7), \mathrm{BA}$ of $54.8 \mathrm{y}( \pm 8.6)$ and $\Delta$ age of $-0.75( \pm 7.72)$. The majority of participants were low-educated $(53.1 \%)$, prevalently never-smokers $(50.0 \%)$ and overweight (42.1\%) (Table 1$)$.

Table 1. Main characteristics of the study population $(n=4952)$ by quartiles of PAC-score.

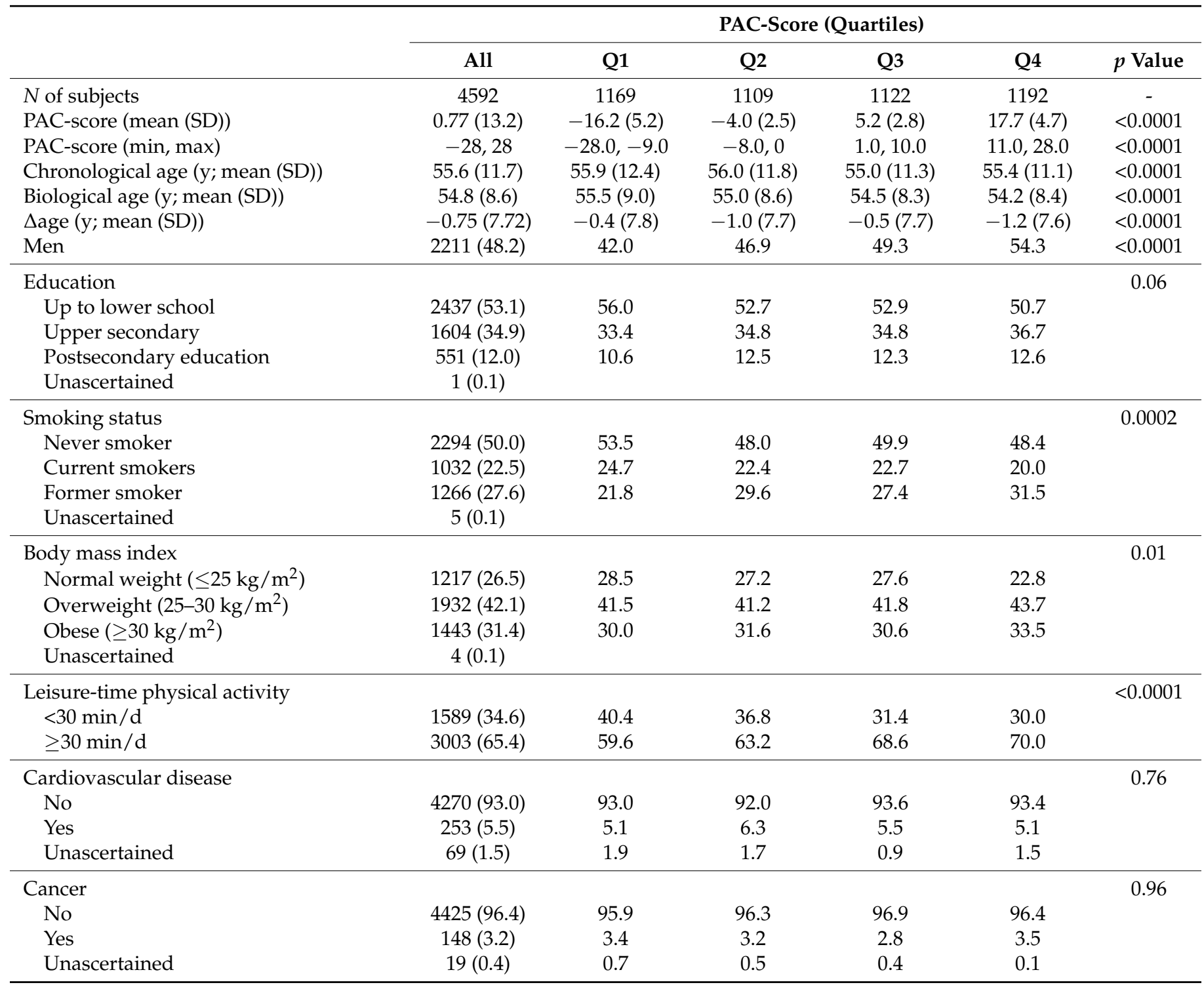


Table 1. Cont.

\begin{tabular}{|c|c|c|c|c|c|c|}
\hline & \multicolumn{6}{|c|}{ PAC-Score (Quartiles) } \\
\hline & All & Q1 & Q2 & Q3 & Q4 & $p$ Value \\
\hline Diabetes & & & & & & 0.42 \\
\hline No & $4305(93.8)$ & 94.3 & 94.2 & 92.7 & 93.8 & \\
\hline Yes & $222(4.8)$ & 4.5 & 4.3 & 5.6 & 4.9 & \\
\hline Unascertained & $65(1.4)$ & 1.2 & 1.4 & 1.7 & 1.3 & \\
\hline Hypertension & & & & & & 0.12 \\
\hline No & $3232(70.4)$ & 69.7 & 69.2 & 70.3 & 72.2 & \\
\hline Yes & $1317(28.7)$ & 29.4 & 29.9 & 28.8 & 26.7 & \\
\hline Unascertained & $43(0.9)$ & 0.9 & 0.9 & 0.9 & 1.1 & \\
\hline Hyperlipidaemia & & & & & & 0.74 \\
\hline No & 4187 (91.2) & 92.1 & 89.6 & 91.9 & 91.0 & \\
\hline Yes & $360(7.8)$ & 7.0 & 9.2 & 7.1 & 8.0 & \\
\hline Unascertained & $45(1.0)$ & 0.9 & 1.2 & 1.0 & 0.9 & \\
\hline Menopausal status & & & & & & 0.95 \\
\hline No & $965(40.5)$ & 41.0 & 39.7 & 41.8 & 40.1 & \\
\hline Yes & $1410(59.2)$ & 59.0 & 60.3 & 58.2 & 60.0 & \\
\hline Unascertained & $6(0.3)$ & & & & & \\
\hline Hormone replacement therapy & & & & & & 0.02 \\
\hline No & $2246(94.3)$ & 96.7 & 94.0 & 92.7 & 93.8 & \\
\hline Yes & $135(5.7)$ & 3.3 & 6.0 & 7.0 & 6.2 & \\
\hline \multicolumn{7}{|l|}{ Polyphenol classes/sub-classes (mg/d) } \\
\hline Flavonols (mean (SD)) & $19.3(10.4)$ & $10.3(4.2)$ & $15.5(4.8)$ & $20.1(5.9)$ & $31.0(10.8)$ & $<0.0001$ \\
\hline Isoflavones (mean (SD)) & $25.2(11.4)$ & $14.5(4.4)$ & $20.8(3.8)$ & $26.3(4.9)$ & $38.8(11.3)$ & $<0.0001$ \\
\hline Lignans (mean (SD)) & $89.4(42.2)$ & $49.2(14.0)$ & $72.3(12.3)$ & $92.6(16.0)$ & $141.8(41.1)$ & $<0.0001$ \\
\hline Flavones (mean (SD)) & $0.8(0.5)$ & $0.5(0.3)$ & $0.7(0.4)$ & $0.9(0.4)$ & $1.2(0.7)$ & $<0.0001$ \\
\hline Flavanones (mean (SD)) & $35.1(17.0)$ & $19.5(7.1)$ & $29.0(6.5)$ & $37.4(9.6)$ & $54.1(17.3)$ & $<0.0001$ \\
\hline Flavanols (mg/d; mean (SD)) & $73.6(83.6)$ & $38.0(70.3)$ & $56.5(52.0)$ & $81.5(70.5)$ & $116.9(106.9)$ & $<0.0001$ \\
\hline Anthocyanidins (mg/d; mean (SD)) & $170.2(104.4)$ & $82.4(42.8)$ & $135.3(57.8)$ & $176.8(67.2)$ & $282.7(107.0)$ & $<0.0001$ \\
\hline \multicolumn{7}{|l|}{ Total antioxidant capacity } \\
\hline FRAP & $19.0(8.6)$ & $15.3(7.2)$ & $17.9(7.7)$ & $19.9(8.2)$ & $22.9(9.3)$ & $<0.0001$ \\
\hline TEAC & $6.3(2.9)$ & $4.9(2.3)$ & $5.9(2.5)$ & $6.6(2.7)$ & $7.7(3.1)$ & $<0.0001$ \\
\hline TRAP & $9.2(4.4)$ & $7.6(3.8)$ & $8.8(4.0)$ & $9.6(4.3)$ & $10.9(4.8)$ & $<0.0001$ \\
\hline Dietary fiber (g/d) & $20.6(7.0)$ & $14.6(3.6)$ & $18.3(3.6)$ & $21.2(4.1)$ & $28.2(7.0)$ & $<0.0001$ \\
\hline Energy intake (kcal/d) & $\begin{array}{l}2113.4 \\
(634.9)\end{array}$ & $\begin{array}{l}1773.3 \\
(512.9)\end{array}$ & $\begin{array}{l}1998.3 \\
(513.8)\end{array}$ & $\begin{array}{l}2181.1 \\
(561.3)\end{array}$ & $\begin{array}{l}2490.5 \\
(693.8)\end{array}$ & $<0.0001$ \\
\hline
\end{tabular}

Values are reported as percentages unless otherwise specified. FRAP $=$ ferric-reducing antioxidant power $\left(\mathrm{mmol} \mathrm{Fe}^{2+}\right)$. TEAC $=$ trolox equivalent antioxidant capacity (mmol Trolox). TRAP = radical-trapping antioxidant parameter (mmol Trolox).

As compared to subjects with lower PAC-score (Q1), those participants reporting higher PAC-score (Q4) had lower $\triangle$ age, were prevalently men, tended to be well-educated and former smokers and practiced more physical exercise, and the women in Q4 were prevalently under replacement hormonal therapy (6.2\%) (Table 1).

Greater intake of polyphenols was also associated with higher antioxidant capacity of the diet and increased fibre and energy intake (Table 1).

In multivariable-adjusted analysis, increased PAC-score was inversely associated with the $\Delta$ age $(\beta=-0.27 ; 95 \% \mathrm{CI}-0.52,-0.02)$. None of the polyphenol classes and sub-classes was associated with $\Delta$ age at the statistical significance thresholds set $(\alpha=0.007)$. Similarly, no association of $\triangle$ age with indices of TAC was observed (Table 2).

Sub-group analyses revealed that the associations of $\Delta$ age with certain polyphenol sub-classes differed by sex; in particular, isoflavones and flavones were inversely associated with $\Delta$ age in women but not in men ( $p$ values for interaction $=0.02$ and 0.0002 , respectively), whereas lignans showed a greater inverse association with $\Delta$ age in women as compared to men $(p$ value for interaction $=0.02)$; finally, flavanols were inversely correlated with 
$\Delta$ age in men $(\beta=-0.09 ; 95 \% \mathrm{CI}-0.32,0.14)$ while being directly associated among women $(\beta=0.17 ; 95 \% \mathrm{CI}-0.06,0.41 ; p$ for interaction $<0.0001)$ (Figure 1).

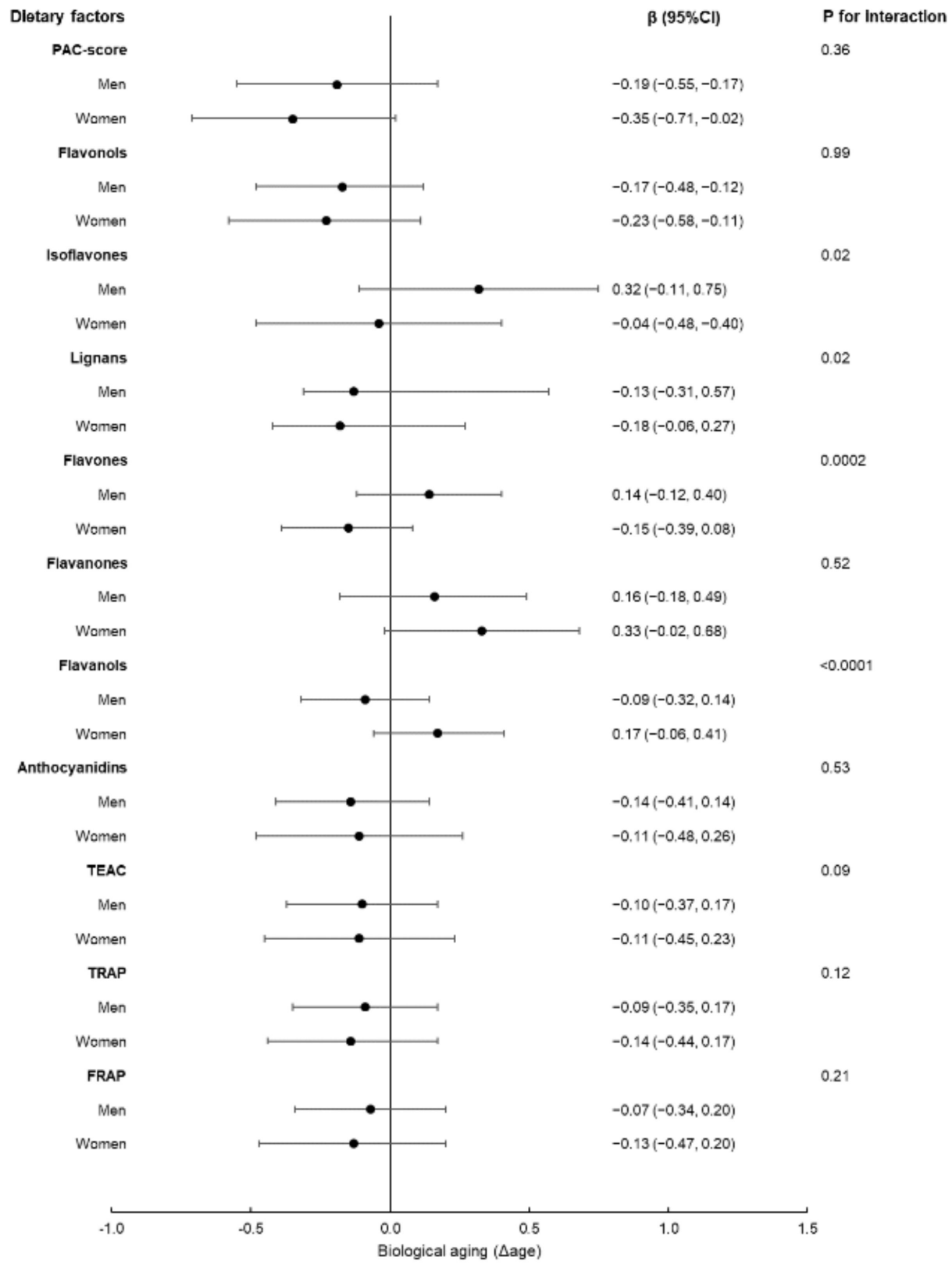

Figure 1. Association of the PAC-score, polyphenol classes and sub-classes and total antioxidant capacity with $\Delta$ age in men $(N=2211)$ and women $(n=2381)$, by means of regression coefficients $(\beta)$ with $95 \%$ confidence interval $(95 \% \mathrm{CI})$. Multivariable-adjusted regression model adjusted for age, sex, energy intake, educational level, leisure-time physical activity, smoking habit, BMI, CVD, cancer, diabetes, hypertension and hyperlipidaemia, menopausal status, hormone replacement therapy and dietary fibre $(\mathrm{g} / \mathrm{d})$. 
Table 2. Association of the PAC-score, polyphenol classes and sub-classes and total antioxidant capacity with $\Delta$ age, by means of regression coefficients $(\beta)$ with $95 \%$ confidence interval $(95 \% \mathrm{CI})$.

\begin{tabular}{|c|c|c|c|c|}
\hline \multirow[b]{2}{*}{ Dietary Factors } & \multicolumn{4}{|c|}{ Biological Aging ( $\Delta$ age) } \\
\hline & $\beta(95 \% \mathrm{CI})^{1}$ & $p$ Value & $\beta(95 \% \mathrm{CI})^{2}$ & $p$ Value \\
\hline PAC-score & $-0.30(-0.49,-0.12)$ & 0.001 & $-0.27(-0.52,-0.02)$ & 0.03 \\
\hline \multicolumn{5}{|c|}{ Polyphenol classes/sub-classes (mg/d) } \\
\hline Flavonols & $-0.28(-0.47,-0.10)$ & 0.003 & $-0.21(-0.44,0.01)$ & 0.06 \\
\hline Isoflavones & $-0.12(-0.30,0.06)$ & 0.18 & $0.13(-0.17,0.44)$ & 0.40 \\
\hline Lignans & $-0.17(-0.35,0.01)$ & 0.06 & $-0.02(-0.34,0.29)$ & 0.89 \\
\hline Flavones & $-0.02(-0.19,0.15)$ & 0.84 & $-0.02(-0.19,0.16)$ & 0.84 \\
\hline Flavanones & $-0.02(-0.20,0.16)$ & 0.82 & $0.23(-0.01,0.47)$ & 0.06 \\
\hline Flavanols & $-0.04(-0.22,0.13)$ & 0.60 & $0.02(-0.14,0.19)$ & 0.78 \\
\hline Anthocyanidins & $-0.25(-0.43,-0.09)$ & 0.05 & $-0.15(-0.37,0.07)$ & 0.17 \\
\hline \multicolumn{5}{|l|}{ Total antioxidant capacity } \\
\hline FRAP & $-0.01(-0.21,0.20)$ & 0.93 & $-0.13(-0.34,0.07)$ & 0.20 \\
\hline TEAC & $-0.03(-0.24,0.17)$ & 0.74 & $-0.14(-0.34,0.07)$ & 0.19 \\
\hline TRAP & $-0.01(-0.20,0.18)$ & 0.94 & $-0.14(-0.34,0.05)$ & 0.16 \\
\hline
\end{tabular}

${ }^{1}$ Regression coefficients (95\%CI) obtained from a model controlled for age, sex and energy intake. ${ }^{2}$ Regression coefficients (95\%CI) obtained from a model controlled for age, sex, energy intake, body mass index, cardiovascular disease, cancer, diabetes, educational level, hormone replacement therapy, hyperlipidaemia, hypertension, leisure-time, menopausal status, physical activity, smoking habit and dietary fiber $(\mathrm{g} / \mathrm{d})$. FRAP $=$ ferric-reducing antioxidant power $\left(\mathrm{mmol} \mathrm{Fe}^{2+}\right) . \mathrm{TEAC}=$ trolox equivalent antioxidant capacity $(\mathrm{mmol}$ Trolox $)$. TRAP = radical-trapping antioxidant parameter (mmol Trolox).

\section{Discussion}

In a large cohort of Italian adults, a diet rich in polyphenols was associated with decelerated biological aging, an emerging predictor of cardiovascular disease risk. Total antioxidant capacity of the diet was not associated with biological age acceleration.

Our results were in line with and extended prior observations from the same cohort, highlighting an inverse association between biological aging and a high adherence to Mediterranean diet which is abundant in polyphenol-rich foods [14].

Consistently, data from other population-based cohorts previously showed a favourable association between polyphenol-rich diets with various markers of aging, including telomere length [25], cognitive decline [35] and DNA methylation [36], that are susceptible to the antioxidant and anti-inflammatory effects of plant-based diets.

Polyphenols are naturally occurring antioxidants mainly present in a large variety of edible plants (i.e., vegetables, cereals, legumes, fruits, nuts, etc.) and plant-derived beverages such as coffee, tea, beer and red wine [19].

Several epidemiological studies have examined the association between intake of certain polyphenol subgroups and their sources, and the incidence of cardiovascular disease [37] and mortality [38]. Moreover, they reported improvements in inflammatory markers [39] and lipid profile associated with increasing polyphenol intake [40].

The health advantages of fruits and vegetables, rich in polyphenols and antioxidants, have been extensively described also in the regulation of inflammation, a major factor contributing to the senescence-and the reduction of-oxidative stress, which plays a key role in the aging process [41-43], and possibly in the etiopathogenesis of osteoporosis [44].

Other studies have also emphasized the impact of diets rich in fruits and vegetables on mitochondrial DNA alterations, implicated in premature aging [23]. Additionally, the benefits of plant-based diets have been studied also with reference to telomere attrition, which is possibly accelerated by oxidative stress and inflammation [25].

Dietary polyphenols likely act on human health through several mechanisms; it has been demonstrated that some polyphenols and their metabolites exert anti-atherosclerotic effects, improve endothelial function and antioxidant status, increase nitric oxide release, and modulate inflammation and lipid metabolism [5,26,31,45-47].

The anti-aging properties of dietary polyphenols have been ascribed at least in part to their antioxidant properties that, through several mechanisms including ROS-scavenging 
actions, contribute to reduce oxidative stress. These mechanisms play an important role in the aging process and various aging-associated chronic diseases [48], including bone mineral density, by preventing oxidation-induced damage to bone cells [49].

Aging is also the result of an imbalance between inflammatory and anti-inflammatory networks [50], a condition often named inflammaging, characterized by elevated levels of blood inflammatory markers that lead to high susceptibility to chronic morbidity, disability, frailty, and premature death [51].

Previous data from the Moli-sani cohort showed an inverse association between dietary polyphenols and a score of low-grade inflammation, which supports the antiinflammatory action of these bioactive compounds [38].

A consistent body of evidence shows a link between dietary intake of antioxidant vitamins and antioxidant-rich foods and lower risk of mortality [52] and cardiovascular disease [53].

As people eat foods in combination, the evaluation of the total antioxidant capacity of the diet is a more valuable approach to measure their joint antioxidant effects [54]. Also, levels of single antioxidants in food do not necessarily reflect their total antioxidant capacity. Rather, this depends on the synergic and redox interactions among the different molecules present in the food [54].

Diets with high antioxidant potential are inversely associated with frailty [55] and with longer telomeres [56], although they were found to be unrelated to risk of major neurological diseases [57].

Of interest, in our study, the absence of an association between total antioxidant capacity of the diet with decelerated biological aging suggests that the observed inverse association of dietary polyphenols with biological aging may not be exclusively due to their antioxidant potential.

Also, because of the relatively poor bioavailability of many of these compounds, their health promoting effects could not be easily explained by the antioxidant action, which may occur only at high circulating and tissue concentrations [58].

Consistently, analyses in the PREDIMED cohort found no association between the antioxidant capacity of the diet and mortality [59], while observing a reduced risk of CVD associated with dietary polyphenols [37]. Similarly, in a cohort of postmenopausal women, dietary polyphenol intake-but not the dietary total antioxidant capacity - was inversely related to cardiovascular disease risk [60], suggesting that mechanisms other than antioxidant activity may be responsible for the health benefits of polyphenols, including positive modulation of gut microbiota [61] and reduction of inflammation [62].

Despite highlighting a relationship between the total content of polyphenols in the diet with decelerated biological aging, we failed to observe an association with the intake of specific polyphenol classes and sub-classes. This could possibly reinforce the actual usefulness of a holistic approach as implemented through the PAC-score.

Some sex-related differences were observed in our study. In particular, we found that dietary polyphenols and some polyphenol sub-classes were more strongly associated with decelerated biological aging among women than in men.

Such sex-based differences in the relation between polyphenols in the diet and health have not been extensively documented [63]. However, a few epidemiological studies revealed that polyphenol intake is more likely to be associated with health advantages among women than in men, as in a recent study showing that polyphenol consumption was significantly associated with individual components of the metabolic syndrome only in women [64]. Similarly, a positive association of total antioxidant capacity of the diet with pulmonary function was observed among women only, being stronger in premenopausal/never smoker women [31]. These sex-related differences may be attributed to physiological differences between men and women, e.g., body composition, gastrointestinal characteristics, renal function and metabolism, that impact on pharmacokinetic parameters [65]. Additionally, a role for sex hormones might explain the differences between men and women [66] in the association of polyphenols and biological aging. Further studies- 
possibly analysing interactions with sex hormone titers-are warranted to investigate this hypothesis.

\section{Strengths and Limitations}

This study has several strengths, including a large sample size with thorough measurement of diet and risk factors to minimize confounding. We used an innovative, machinelearning-based measure of biological aging; a deep neural network was applied to a large number of circulating biomarkers, an approach which has, to date, been developed in just a few cohorts worldwide [8,67].

However, there are some limitations that need to be carefully considered. First, the observational nature of the study could not fully rule out residual or unmeasured confounding. In addition, the present findings were derived from a cross-sectional analysis, with consequent difficulty to define directionality of effect. Second, dietary data were based on self-reported information collected through the use of an FFQ and therefore may be susceptible to misreporting that could have led to measurement error and bias or to difficulties in assessing portion sizes and subsequent inadequacies in food composition tables. However, such potential limitations were mitigated by energy adjustment. Also, the validated FFQ used in this study has been previously largely employed and has been reported to have predictive validity for a number of health outcomes [61,62]. Third, our data were obtained from a cohort of adults residing in a small Southern Italian region, and this might limit the generalizability of our findings, although the main characteristics of our population are comparable to those of the Italian Cardiovascular Epidemiological Observatory and are therefore representative of at least the Italian population [68]. Finally, other assessments of biological aging might show a higher accuracy than blood age, e.g., brain age [10], but these are based on expensive instrumental assessments (i.e., Magnetic Resonance Imaging) and not easily applicable to large population-based cohorts.

\section{Conclusions}

Results from this Mediterranean cohort indicate that a diet rich in polyphenols is associated with decelerated biological aging, a novel predictor of cardiovascular disease risk, possibly through mechanisms that go beyond their antioxidant activity.

To the best of our knowledge, this is the first population-based study addressing the association between polyphenol-rich diets and biological aging as reflected by a machinelearning-based measure of blood-based biological age.

Our results have added to the existing literature by expanding knowledge on the anti-aging properties of dietary polyphenols. Future longitudinal analyses are warranted to confirm our results and to test whether a favourable modulation of biological aging is likely to mediate the well-established relationship between polyphenol-rich diets and risk of cardiovascular disease-and possibly of bone health.

Author Contributions: L.I., M.B., S.E. and A.G. conceived the present study, contributed to its design and to interpretation of data; S.C., A.G., E.R. and M.P. managed data collection; A.D.C. (Amalia De Curtis) performed laboratory tests; A.D.C. (Augusto Di Castelnuovo), S.E. and A.G. analysed the data; S.E. and M.B. wrote the manuscript; M.B.D., C.C., G.d.G. and L.I. originally inspired the Moli-sani study and critically reviewed the manuscript. All authors have read and agreed to the published version of the manuscript.

Funding: The original enrolment phase of the Moli-sani Study (2005-2010) was supported by research grants from Pfizer Foundation (Rome, Italy), the Italian Ministry of University and Research (MIUR, Rome, Italy)-Programma Triennale di Ricerca, Decreto no.1588 and Instrumentation Laboratory, Milan, Italy. This work was partially supported by the Italian Ministry of Economic Development (bando PON I\&C 2014-2020; PLATONE Project - Platform for Integrated Health Life - n. F/080032/0103/X35) and by the Italian Ministry of Health (grant RF-2018-12367074 to G.d.G. and S.C.). Funders had no role in study design, collection, analysis, or interpretation of data, nor in the writing of the manuscript, nor in the decision to submit this article for publication. All authors were and are independent from funders. 
Institutional Review Board Statement: The study was conducted according to the guidelines of the Declaration of Helsinki, and approved by the Ethics Committee of the Catholic University of the Sacred Heart in Rome (protocol code 99; 8/03/2004).

Informed Consent Statement: Informed consent was obtained from all subjects involved in the study.

Data Availability Statement: The data sets analysed in the current study are not publicly available because of restricted access, but further information about the data sets is available from the corresponding author on reasonable request.

Acknowledgments: We are grateful to the population of the Molise region who enthusiastically joined the study and to the Associazione Cuore Sano ONLUS (Campobasso, Italy) for its support to the research activities. E.R. and A.G. are supported by Fondazione Umberto Veronesi, which is gratefully acknowledged. The enrolment phase of the Moli-sani Study was conducted at the Research Laboratories of the Catholic University in Campobasso (Italy). The follow up of the Moli-sani cohort is being conducted at the Department of Epidemiology and Prevention of the IRCCS Neuromed, Pozzilli, Italy. Steering Committee: Licia Iacoviello *o (Chairperson), Giovanni de Gaetano * and Maria Benedetta Donati *. Scientific secretariat: Marialaura Bonaccio *, Americo Bonanni *, Chiara Cerletti *, Simona Costanzo *, Amalia De Curtis *, Augusto Di Castelnuovo §, Francesco Gianfagna ${ }^{\circ} \S$, Mariarosaria Persichillo *, Teresa Di Prospero * (Secretary). Safety and Ethics Committee: Jos Vermylen (Catholic Univesity, Leuven, Belgio) (Chairperson), Ignacio De Paula Carrasco (Accademia Pontificia Pro Vita, Roma, Italy), Antonio Spagnuolo (Catholic University, Roma, Italy). External Event adjudicating Committee: Deodato Assanelli (Brescia, Italy), Vincenzo Centritto (Campobasso, Italy). Baseline and Follow-up data management: Simona Costanzo * (Coordinator), Marco Olivieri (Associazione Cuore Sano, Campobasso, Italy), Teresa Panzera * Data Analysis: Augusto Di Castelnuovo § (Coordinator), Marialaura Bonaccio *, Simona Costanzo *, Simona Esposito *, Alessandro Gialluisi *, Francesco Gianfagna ${ }^{\circ} \S$, Emilia Ruggiero *. Biobank and biochemical laboratory: Amalia De Curtis * (Coordinator), Sara Magnacca §. Genetic laboratory: Benedetta Izzi * (Coordinator), Annalisa Marotta *, Fabrizia Noro *, Roberta Parisi *, Alfonsina Tirozzi * Recruitment staff: Mariarosaria Persichillo * (Coordinator), Francesca Bracone *, Francesca De Lucia (Associazione Cuore Sano, Campobasso, Italy), Cristiana Mignogna ${ }^{\circ}$, Teresa Panzera *, Livia Rago *. Communication and Press Office: Americo Bonanni *. Regional Health Institutions: Direzione Generale per la Salute-Regione Molise; Azienda Sanitaria Regionale del Molise (ASReM, Italy); Molise Dati Spa (Campobasso, Italy); Offices of vital statistics of the Molise region. Hospitals: Presidi Ospedalieri ASReM: Ospedale A. Cardarelli-Campobasso, Ospedale F. Veneziale—Isernia, Ospedale San Timoteo-Termoli (CB), Ospedale Ss. Rosario-Venafro (IS), Ospedale Vietri-Larino (CB), Ospedale San Francesco Caracciolo-Agnone (IS); Casa di Cura Villa Maria-Campobasso; Ospedale Gemelli Molise-Campobasso; IRCCS Neuromed-Pozzilli (IS). * Department of Epidemiology and Prevention, IRCCS Neuromed, Pozzilli, Italy. ${ }^{\circ}$ Department of Medicine and Surgery, University of Insubria, Varese, Italy. § Mediterranea Cardiocentro, Napoli, Italy. Baseline Recruitment staff is available at https:/ / www.moli-sani.org/?page_id=173.

Conflicts of Interest: None of the authors of the present manuscript have conflicts of interest to disclose.

\section{References}

1. World Health Organization. Life Expectancy. 2016. Available online: http://www.who.int/gho/mortality_burden_disease/life_ tables/situation_trends/en/ (accessed on 9 November 2017).

2. Karasik, D.; Demissie, S.; Cupples, L.A.; Kiel, D.P. Disentangling the genetic determinants of human aging: Biological age as an alternative to the use of survival measures. J. Gerontol. Ser. A Biol. Sci. Med Sci. 2005, 60, 574-587. [CrossRef]

3. Cohen, J.E. Human Population: The Next Half Century. Science 2003, 302, 1172-1175. [CrossRef]

4. De Lucia, C.; Murphy, T.; Steves, C.J.; Dobson, R.J.B.; Proitsi, P.; Thuret, S. Lifestyle mediates the role of nutrient-sensing pathways in cognitive aging: Cellular and epidemiological evidence. Commun. Biol. 2020, 3, 1-17. [CrossRef]

5. Han, K.-T.; Kim, D.W.; Kim, S.J.; Kim, S.J. Biological Age Is Associated with the Active Use of Nutrition Data. Int. J. Environ. Res. Public Health 2018, 15, 2431. [CrossRef] [PubMed]

6. Finkel, D.; Whitfield, K.; McGue, M. Genetic and Environmental Influences on Functional Age: A Twin Study. J. Gerontol. Ser. B 1995, 50, P104-P113. [CrossRef]

7. Gialluisi, A.; Di Castelnuovo, A.; Donati, M.B.; De Gaetano, G.; Iacoviello, L.; the Moli-sani Study Investigators. Machine Learning Approaches for the Estimation of Biological Aging: The Road Ahead for Population Studies. Front. Med. 2019,6 , 146. [CrossRef] [PubMed] 
8. Mamoshina, P.; Kochetov, K.; Putin, E.; Cortese, F.; Aliper, A.; Lee, W.-S.; Ahn, S.-M.; Uhn, L.; Skjodt, N.; Kovalchuk, O.; et al. Population Specific Biomarkers of Human Aging: A Big Data Study Using South Korean, Canadian, and Eastern European Patient Populations. J. Gerontol. Ser. A Biol. Sci. Med. Sci. 2018, 73, 1482-1490. [CrossRef]

9. Klemera, P.; Doubal, S. A new approach to the concept and computation of biological age. Mech. Ageing Dev. 2006, 127, 240-248. [CrossRef]

10. Fiorito, G.; Polidoro, S.; Dugue, P.-A.; Kivimaki, M.; Ponzi, E.; Matullo, G.; Guarrera, S.; Assumma, M.B.; Georgiadis, P.; Kyrtopoulos, S.A.; et al. Social adversity and epigenetic aging: A multi-cohort study on socioeconomic differences in peripheral blood DNA methylation. Sci. Rep. 2017, 7, 1-12. [CrossRef]

11. Park, J.; Cho, B.; Kwon, H.; Lee, C. Developing a biological age assessment equation using principal component analysis and clinical biomarkers of aging in Korean men. Arch. Gerontol. Geriatr. 2009, 49, 7-12. [CrossRef]

12. Cole, J.H.; Franke, K. Predicting Age Using Neuroimaging: Innovative Brain Ageing Biomarkers. Trends Neurosci. 2017, 40, 681-690. [CrossRef] [PubMed]

13. Levine, M.E. Modeling the Rate of Senescence: Can Estimated Biological Age Predict Mortality More Accurately Than Chronological Age? J. Gerontol. Ser. A Biol. Sci. Med. Sci. 2013, 68, 667-674. [CrossRef]

14. Gialluisi, A.; Di Castelnuovo, A.; Costanzo, S.; Bonaccio, M.; Magnacca, S.; De Curtis, A.; Cerletti, C.; Donati, M.B.; de Gaetano, G.; Capobianco, E.; et al. Exploring domains, clinical implications and environmental asso-ciations of a deep learning marker of biological ageing. medRxiv 2021. [CrossRef]

15. Levine, M.; Crimmins, E. Evidence of accelerated aging among African Americans and its implications for mortality. Soc. Sci. Med. 2014, 118, 27-32. [CrossRef]

16. Yoo, J.; Kim, Y.; Cho, E.R.; Jee, S.H. Biological age as a useful index to predict seventeen-year survival and mortality in Koreans. BMC Geriatr. 2017, 17, 7. [CrossRef] [PubMed]

17. Mamoshina, P.; Kochetov, K.; Cortese, F.; Kovalchuk, A.; Aliper, A.; Putin, E.; Scheibye-Knudsen, M.; Cantor, C.R.; Skjodt, N.M.; Kovalchuk, O.; et al. Blood Biochemistry Analysis to Detect Smoking Status and Quantify Accelerated Aging in Smokers. Sci. Rep. 2019, 9, 142. [CrossRef]

18. Pyrkov, T.V.; Fedichev, P.O. Biological age is a universal marker of aging, stress, and frailty. bioRxiv 2019. [CrossRef]

19. Scalbert, A.; Johnson, I.T.; Saltmarsh, M. Polyphenols: Antioxidants and beyond. Am. J. Clin. Nutr. 2005, 81, 215S-217S. [CrossRef]

20. Silva, R.F.M.; Pogačnik, L. Polyphenols from Food and Natural Products: Neuroprotection and Safety. Antioxidants $2020,9,61$. [CrossRef] [PubMed]

21. Movassagh, E.Z.; Vatanparast, H. Current Evidence on the Association of Dietary Patterns and Bone Health: A Scoping Review. Adv. Nutr. 2017, 8, 1-16. [CrossRef] [PubMed]

22. García-Gavilán, J.; Bulló, M.; Canudas, S.; Martínez-González, M.; Estruch, R.; Giardina, S.; Fitó, M.; Corella, D.; Ros, E.; Salas-Salvadó, J. Extra virgin olive oil consumption reduces the risk of osteoporotic fractures in the PREDIMED trial. Clin. Nutr. 2018, 37, 329-335. [CrossRef] [PubMed]

23. Wu, S.; Li, X.; Meng, S.; Fung, T.; Chan, A.T.; Liang, G.; Giovannucci, E.; De Vivo, I.; Lee, J.H.; Nan, H. Fruit and vegetable consumption, cigarette smoke, and leukocyte mitochondrial DNA copy number. Am. J. Clin. Nutr. 2019, 109, 424-432. [CrossRef] [PubMed]

24. Meinilä, J.; Perälä, M.-M.; Kautiainen, H.; Männistö, S.; Kanerva, N.; Shivappa, N.; Hébert, J.R.; Iozzo, P.; Guzzardi, M.A.; Eriksson, J.G. Healthy diets and telomere length and attrition during a 10-year follow-up. Eur. J. Clin. Nutr. 2019, 73, 1352-1360. [CrossRef] [PubMed]

25. Crous-Bou, M.; Molinuevo, J.-L.; Sala-Vila, A. Plant-Rich Dietary Patterns, Plant Foods and Nutrients, and Telomere Length. Adv. Nutr. 2019, 10, S296-S303. [CrossRef]

26. Brighenti, F.; Valtueña, S.; Pellegrini, N.; Ardigò, D.; Del Rio, D.; Salvatore, S.; Piatti, P.; Serafini, M.; Zavaroni, I. Total antioxidant capacity of the diet is inversely and independently related to plasma concentration of high-sensitivity C-reactive protein in adult Italian subjects. Br. J. Nutr. 2005, 93, 619-625. [CrossRef]

27. Iacoviello, L.; Bonanni, A.; Costanzo, S.; De Curtis, A.; Di Castelnuovo, A.; Olivieri, M.; Zito, F.; Donati, M.B.; de Gaetano, G. The Moli-Sani Project, a randomized, prospective cohort study in the Molise region in Italy; design, rationale and objectives. Ital. J. Public Health 2007, 4, 110-118. [CrossRef]

28. Pisani, P.; Faggiano, F.; Krogh, V.; Palli, D.; Vineis, P.; Berrino, F. Relative validity and reproducibility of a food frequency dietary questionnaire for use in the Italian EPIC centres. Int. J. Epidemiol. 1997, 26, S152-S160. [CrossRef]

29. Pala, V.; Sieri, S.; Palli, D.; Salvini, S.; Berrino, F.; Bellegotti, M.; Frasca, G.; Tumino, R.; Sacerdote, C.; Fiorini, L.; et al. Diet in the Italian Epic Cohorts: Presentation of Data and Methodological Issues. Tumori J. 2003, 89, 594-607. [CrossRef]

30. Italian Food Composition Tables. Istituto Nazionale di Ricerca per gli Alimenti e la Nutrizione. Available online: http: //www.inran.it/646/tabelle_di_composizione_degli_alimenti.html (accessed on 1 May 2015).

31. Di Giuseppe, R.; Arcari, A.; Serafini, M.; Di Castelnuovo, A.; Zito, F.; De Curtis, A.; Sieri, S.; Krogh, V.; Pellegrini, N.; Schünemann, H.J.; et al. Total dietary antioxidant capacity and lung function in an Italian population: A favorable role in premenopausal/never smoker women. Eur. J. Clin. Nutr. 2011, 66, 61-68. [CrossRef] [PubMed]

32. Pounis, G.D.; Di Castelnuovo, A.; Bonaccio, M.; Costanzo, S.; Persichillo, M.; Krogh, V.; Donati, M.B.; De Gaetano, G.; Iacoviello, L. Flavonoid and lignan intake in a Mediterranean population: Proposal for a holistic approach in polyphenol dietary analysis, the Moli-sani Study. Eur. J. Clin. Nutr. 2015, 70, 338-345. [CrossRef] 
33. BioActive Substances in Food Information System, eBASIS. Eurofir. Available online: http://ebasis.eurofir.org/Default.asp (accessed on 1 March 2015).

34. Pellegrini, N.; Salvatore, S.; Valtueña, S.; Bedogni, G.; Porrini, M.; Pala, V.; Del Rio, D.; Sieri, S.; Miglio, C.; Krogh, V.; et al. Development and Validation of a Food Frequency Questionnaire for the Assessment of Dietary Total Antioxidant Capacity. J. Nutr. 2007, 137, 93-98. [CrossRef] [PubMed]

35. Elliott, M.L.; Belsky, D.W.; Knodt, A.R.; Ireland, D.; Melzer, T.R.; Poulton, R.; Ramrakha, S.; Caspi, A.; Moffitt, T.E.; Hariri, A.R. Brain-age in midlife is associated with accelerated biological aging and cognitive decline in a longitudinal birth cohort. Mol. Psychiatry 2019, 10. [CrossRef] [PubMed]

36. Hibler, E.; Huang, L.; Andrade, J.; Spring, B. Impact of a diet and activity health promotion intervention on regional patterns of DNA methylation. Clin. Epigenet. 2019, 11, 133. [CrossRef] [PubMed]

37. Tresserra-Rimbau, A.; Rimm, E.; Medina-Remón, A.; Martínez-González, M.; de la Torre, R.; Corella, D.; Salas-Salvadó, J.; GómezGracia, E.; Lapetra, J.; Arós, F.; et al. Inverse association between habitual polyphenol intake and incidence of cardiovascular events in the PREDIMED study. Nutr. Metab. Cardiovasc. Dis. 2014, 24, 639-647. [CrossRef] [PubMed]

38. Pounis, G.; Costanzo, S.; Bonaccio, M.; Di Castelnuovo, A.; De Curtis, A.; Ruggiero, E.; Persichillo, M.; Cerletti, C.; Donati, M.B.; De Gaetano, G.; et al. Reduced mortality risk by a polyphenol-rich diet: An analysis from the Moli-sani study. Nutrition 2018, 48, 87-95. [CrossRef] [PubMed]

39. Estruch, R.; Sacanella, E.; Badia, E.; Antúnez, E.; Nicolás, J.M.; Fernández-Solá, J.; Rotilio, D.; de Gaetano, G.; Rubin, E.; UrbanoMárquez, A. Different effects of red wine and gin consumption on inflammatory biomarkers of atherosclerosis: A prospective randomized crossover trial. Atherosclerosis 2004, 175, 117-123. [CrossRef]

40. Speer, H.; D’Cunha, N.M.; Botek, M.; McKune, A.J.; Sergi, D.; Georgousopoulou, E.; Mellor, D.D.; Naumovski, N. The Effects of Dietary Polyphenols on Circulating Cardiovascular Disease Biomarkers and Iron Status: A Systematic Review. Nutr. Metab. Insights 2019, 12. [CrossRef] [PubMed]

41. Queen, B.L.; Tollefsbol, T.O. Polyphenols and Aging. Curr. Aging Sci. 2010, 3, 34-42. [CrossRef]

42. Chrysohoou, C.; Stefanadis, C. Longevity and Diet. Myth or pragmatism? Maturitas 2013, 76, 303-307. [CrossRef]

43. Serino, A.; Salazar, G. Protective Role of Polyphenols against Vascular Inflammation, Aging and Cardiovascular Disease. Nutrition 2018, 11, 53. [CrossRef]

44. Barbour, K.E.; Boudreau, R.; Danielson, M.E.; Youk, A.O.; Wactawski-Wende, J.; Greep, N.C.; Lacroix, A.Z.; Jackson, R.D.; Wallace, R.B.; Bauer, D.C.; et al. Inflammatory markers and the risk of hip fracture: The women's health initiative. J. Bone Miner. Res. 2012, 27, 1167-1176. [CrossRef] [PubMed]

45. Quach, A.; Levine, M.E.; Tanaka, T.; Lu, A.T.; Chen, B.H.; Ferrucci, L.; Ritz, B.; Bandinelli, S.; Neuhouser, M.L.; Beasley, J.M.; et al. Epigenetic clock analysis of diet, exercise, education, and lifestyle factors. Aging 2017, 9, 419-446. [CrossRef]

46. Pounis, G.; Costanzo, S.; Di Giuseppe, R.; De Lucia, F.; Santimone, I.; Sciarretta, A.; Barisciano, P.; Persichillo, M.; De Curtis, A.; Zito, F.; et al. Consumption of healthy foods at different content of antioxidant vitamins and phytochemicals and metabolic risk factors for cardiovascular disease in men and women of the Moli-sani study. Eur. J. Clin. Nutr. 2012, 67, 207-213. [CrossRef] [PubMed]

47. Flanagan, E.W.; Most, J.; Mey, J.T.; Redman, L.M. Calorie Restriction and Aging in Humans. Annu. Rev. Nutr. 2020, 40, 105-133. [CrossRef] [PubMed]

48. Luo, J.; Si, H.; Jia, Z.; Liu, D. Dietary Anti-Aging Polyphenols and Potential Mechanisms. Antioxidants 2021, 10, 283. [CrossRef]

49. Chisari, E.; Shivappa, N.; Vyas, S. Polyphenol-Rich Foods and Osteoporosis. Curr. Pharm. Des. 2019, 25, 2459-2466. [CrossRef] [PubMed]

50. Franceschi, C.; Capri, M.; Monti, D.; Giunta, S.; Olivieri, F.; Sevini, F.; Panourgia, M.P.; Invidia, L.; Celani, L.; Scurti, M.; et al. Inflammaging and anti-inflammaging: A systemic perspective on aging and longevity emerged from studies in humans. Mech. Ageing Dev. 2007, 128, 92-105. [CrossRef] [PubMed]

51. Ferrucci, L.; Fabbri, E. Inflammageing: Chronic inflammation in ageing, cardiovascular disease, and frailty. Nat. Rev. Cardiol. 2018, 15, 505-522. [CrossRef]

52. Zhao, L.-G.; Shu, X.-O.; Li, H.-L.; Zhang, W.; Gao, J.; Sun, J.-W.; Zheng, W.; Xiang, Y.-B. Dietary antioxidant vitamins intake and mortality: A report from two cohort studies of Chinese adults in Shanghai. J. Epidemiol. 2017, 27, 89-97. [CrossRef]

53. Kubota, Y.; Iso, H.; Date, C.; Kikuchi, S.; Watanabe, Y.; Wada, Y.; Inaba, Y.; Tamakoshi, A.; THE JACC Study Group. Dietary Intakes of Antioxidant Vitamins and Mortality from Cardiovascular Disease. Stroke 2011, 42, 1665-1672. [CrossRef]

54. Pellegrini, N.; Serafini, M.; Colombi, B.; Del Rio, D.; Salvatore, S.; Bianchi, M.; Brighenti, F. Total Antioxidant Capacity of Plant Foods, Beverages and Oils Consumed in Italy Assessed by Three Different In Vitro Assays. J. Nutr. 2003, 133, 2812-2819. [CrossRef]

55. Kobayashi, S.; Asakura, K.; Suga, H.; Sasaki, S.; Three-generation Study of Women on Diets and Health Study Groups. Inverse association between dietary habits with high total antioxidant capacity and prevalence of frailty among elderly Japanese women: A multicenter cross-sectional study. J. Nutr. Health Aging 2014, 18. [CrossRef]

56. García-Calzón, S.; Moleres, A.; Martínez-González, M.A.; Martínez, J.A.; Zalba, G.; Marti, A. Dietary total antioxidant capacity is associated with leukocyte telomere length in a children and adolescent population. Clin. Nutr. 2015, 34, 694-699. [CrossRef] [PubMed] 
57. Devore, E.E.; Feskens, E.; Ikram, M.A.; Heijer, T.D.; Vernooij, M.; Van Der Lijn, F.; Hofman, A.; Niessen, W.J.; Breteler, M.M. Total antioxidant capacity of the diet and major neurologic outcomes in older adults. Neurology 2013, 80, 904-910. [CrossRef]

58. Cipolletti, M.; Fernandez, V.S.; Montalesi, E.; Marino, M.; Fiocchetti, M. Beyond the Antioxidant Activity of Dietary Polyphenols in Cancer: The Modulation of Estrogen Receptors (ERs) Signaling. Int. J. Mol. Sci. 2018, 19, 2624. [CrossRef] [PubMed]

59. Henríquez-Sánchez, P.; Sanchez-Villegas, A.; Ruano-Rodríguez, C.; Gea, A.; Lamuela-Raventos, R.M.; Estruch, R.; Salas-Salvadó, J.; Covas, M.I.; Corella, D.; Schroder, H.; et al. Dietary total antioxidant capacity and mortality in the PREDIMED study. Eur. J. Nutr. 2015, 55, 227-236. [CrossRef] [PubMed]

60. Witkowska, A.M.; Waśkiewicz, A.; Zujko, M.E.; Szcześniewska, D.; Pająk, A.; Stepaniak, U.; Drygas, W. Dietary Polyphenol Intake, but Not the Dietary Total Antioxidant Capacity, Is Inversely Related to Cardiovascular Disease in Postmenopausal Polish Women: Results of WOBASZ and WOBASZ II Studies. Oxidative Med. Cell. Longev. 2017, 2017, 5982809. [CrossRef]

61. Cardona, F.; Andrés-Lacueva, C.; Tulipani, S.; Tinahones, F.J.; Queipo-Ortuño, M.I. Benefits of polyphenols on gut microbiota and implications in human health. J. Nutr. Biochem. 2013, 24, 1415-1422. [CrossRef]

62. Wu, M.; Luo, Q.; Nie, R.; Yang, X.; Tang, Z.; Chen, H. Potential implications of polyphenols on aging considering oxidative stress, inflammation, autophagy, and gut microbiota. Crit. Rev. Food Sci. Nutr. 2020. [CrossRef]

63. Del Bo', C.; Bernardi, S.; Marino, M.; Porrini, M.; Tucci, M.; Guglielmetti, S.; Cherubini, A.; Carrieri, B.; Kirkup, B.; Kroon, P.; et al. Systematic Review on Polyphenol Intake and Health Outcomes: Is there Sufficient Evidence to Define a Health-Promoting Polyphenol-Rich Dietary Pattern? Nutrition 2019, 11, 1355. [CrossRef]

64. Grosso, G.; Stepaniak, U.; Micek, A.; Stefler, D.; Bobak, M.; Pająk, A. Dietary polyphenols are inversely associated with metabolic syndrome in Polish adults of the HAPIEE study. Eur. J. Nutr. 2017, 56, 1409-1420. [CrossRef] [PubMed]

65. Franconi, F.; Brunelleschi, S.; Steardo, L.; Cuomo, V. Gender differences in drug responses. Pharmacol. Res. 2007, 55, 81-95. [CrossRef] [PubMed]

66. Rosano, G.M.C.; Spoletini, I.; Vitale, C. Cardiovascular disease in women, is it different to men? The role of sex hormones. Climacteric 2017, 20, 125-128. [CrossRef] [PubMed]

67. Putin, E.; Mamoshina, P.; Aliper, A.; Korzinkin, M.; Moskalev, A.; Kolosov, A.; Ostrovskiy, A.; Cantor, C.; Vijg, J.; Zhavoronkov, A. Deep biomarkers of human aging: Application of deep neural networks to biomarker development. Aging 2016, 8, 1021-1033. [CrossRef] [PubMed]

68. Palmieri, L.; Rielli, R.; Demattè, L.; Donfrancesco, C.; Ciccarelli, P.; Caiola, P.D.S.; Dima, F.; Noce, C.L.; Brignoli, O.; Cuffari, A.; et al. CUORE project: Implementation of the 10-year risk score. Eur. J. Cardiovasc. Prev. Rehabil. 2011, 18, 642-649. [CrossRef] [PubMed] 